\title{
Dynamic psychiatry and the psychodynamic formulation
}

\author{
MW Böhmer \\ Department of Psychiatry, University of Pretoria, Weskoppies Hospital, Pretoria, South Africa
}

\begin{abstract}
This article aims to describe the difference between descriptive and dynamic psychiatry. As part of the latter every psychiatrist should be able to construct a psychodynamic formulation. A psychodynamic formulation, an indication of psychological mindedness, helps the psychiatrist to recognize the unique, personal aspects of the illness and the life of the patient. The formulation furthermore helps with the initial orientation towards the patient: it anticipates and predicts how the patient might interact with the psychiatrist and how defenses and underlying conflicts will manifest themselves in treatment. The formulation can therefore be very useful in the treatment of any patient, since it can help to understand and manage the patient's attitude towards being prescribed medication, as well as compliance and prognosis and serve as an initial guide in the psychotherapy. The psychodynamic formulation is unfortunately seldom incorporated in the psychiatric presentation of patients; guidelines are therefore given to especially help psychiatric trainees with the construction of such a formulation.
\end{abstract}

Keywords: Dynamic psychiatry; Psychodynamic formulation; Psychiatric training

Received: 16-10-2010

Accepted: $23-11-2010$

doi: http://dx.doi.org/10.4314/ajpsy.v14i4.3

\section{Introduction}

In her book 'An Unquiet Mind' the psychologist Kay Jamison gives a moving account of her suffering from a bipolar mood disorder. ${ }^{1}$ About her treatment she writes: "At this point in my existence, I cannot imagine leading a normal life without both taking lithium and having had the benefits of psychotherapy. Lithium prevents my seductive but disastrous highs, diminishes my depressions, clears out the wool and webbing from my disordered thinking, slows me down, gentles me out, keeps me from ruining my career and relationships, keeps me out of a hospital, alive, and makes psychotherapy possible. But, ineffably, psychotherapy heals. It makes some sense of the confusion, reins in the terrifying thoughts and feelings, returns some control and hope and possibility of learning from it all."

For Jamison it is not to be questioned that medication and psychotherapy go hand in hand: mind and brain cannot be separated. In her regular, at least once a week sessions with her psychiatrist, the despair that at times threatened to overwhelm her was contained. Her psychiatrist, in his integrative approach of prescribing medication and providing psychotherapy, helped her to live a productive and meaningful life.

\section{Correspondence}

Dr. MW Böhmer

Weskoppies Hospital

Private Bag X 113, Pretoria, 0001, South Africa

email: mwbohmer@absamail.co.za
Cartesian dualism has been very influential and potentially pernicious in the field of psychiatry. ${ }^{2}$ Kendler states that we need to reject the belief that mind and brain reflect two fundamentally different entities. Although both mental processes and psychiatric disorders are biological, the range of causal processes that are operative in psychiatric illness cannot be explained by biological reductionism, which could be seen as a logical consequence of this line of thinking. ${ }^{2}$ Life events, cultural processes and other factors influence and modify gene expression, brain anatomy and functioning. 2,3 Changes in the brain affect the mind, but what happens in the mind also affects the brain. Pharmacotherapy and psychotherapy may both lead to changes in the brain. ${ }^{3}$

Kandel, the eminent psychiatrist and recipient of the Nobel Prize in 2000 for his neuro-scientific research, writes that psychoanalysis has revolutionized our understanding of mental life. It has provided remarkable insights about the functioning of the psyche and "still represents the most coherent and intellectually satisfying view of the mind". ${ }^{3,4} \mathrm{He}$ also writes that psychoanalysis has unfortunately not evolved scientifically and has not integrated and incorporated into its view of the mind "the rich harvest of knowledge about the biology of the brain and its control over behavior that has emerged in the last 50 years". ${ }^{4}$ Kandel pleads, following Freud, for a psychiatry in which biology is integrated with the rich insights that have come from psychoanalysis, in which mind and brain are each given their appropriate place. Psychoanalytic psychiatry, because of "the deep concern of psychoanalysis for the 
integrity of an individual's personal history", has "helped develop direct and respectful ways for physicians to interact with mentally ill patients". 3

According to Browning "many modern psychotherapists are aware that respect for persons gets to the heart of psychological cure". He states that respect can only become restorative if it is shown concretely with reference to that person's specific story and not just to the abstract person. ${ }^{5}$ The importance of the patient's story is also emphasized by the following comments made by a despondent physician who had just been interviewed by a psychiatrist ${ }^{6}$ "I don't think he heard me ... Depression may be the disease, but it is not the problem. The problem is my life. .... It's falling apart. My marriage. My relationship with my kids. My confidence in my research. My sense of purpose. My dreams. Is this depression? ... I want this depression treated, all right. There is something more I want, however. I want to tell this story, my story. I want someone trained to hear me. I thought that was what psychiatrists did."

Dynamic psychiatry aims to do just this - furthering interest and respect for the patient's unique, specific story. Wallace, arguing in favor of dynamic psychiatry, describes it as the most holistic model in psychiatry. ${ }^{7} \mathrm{He}$ considers the dynamic model the "useable core" of Freud's teachings, embellished by interpersonal theories (e.g. Sullivan) and object relations theory. Dynamic psychiatry, he writes, is not antagonistic to the data of biological psychiatry, social psychiatry, systems approaches or behavioral psychology. It is interested and incorporates data from all of them. The dynamic model "appreciates, better than any other, that human behavior is an epiphenomenon of neither culture nor biology, but of an interaction between the two". ${ }^{7}$ Dynamic psychiatry is described by Gabbard as "an approach to diagnosis and treatment characterized by a way of thinking about both patient and clinician that includes unconscious conflict, deficits and distortions of intra-psychic structures, and internal object relations and that integrates these elements with contemporary findings from the neurosciences". ${ }^{8}$ In his definition the personal history of the patient is emphasized, as well as the interaction between clinician and patient.

Neurobiology is also included; there is no antagonism towards biological aspects of mental illness. Both Wallace and Gabbard use the terms dynamic or psychodynamic psychiatry interchangeably.

Ultimately, however, no theory or formulation will guarantee a respectful attitude; respect will depend on the doctor's attitude towards the patient.

\section{Differentiating descriptive from dynamic psychiatry}

Descriptive psychiatry categorizes patients according to clusters of symptoms, as well as common behavioral and phenomenological features. ${ }^{8}$ The patient's subjective experience is less important. An example would be diagnosing a patient with a Major Depressive Disorder according to DSM IV, searching thus for those features that a patient has in common with others suffering from a similar problem and to then provide a summary label. ${ }^{9}$ The diagnosis in descriptive psychiatry cannot indicate the complexity of the different factors and chain of events leading to the development of a mental illness. ${ }^{10}$

Dynamic psychiatry encompasses descriptive psychiatry, but is furthermore interested in the subjective and personal experiences of the patient. Dynamic psychiatry fosters a basic psychological mindedness; a patient is not just seen as someone with a descriptive diagnosis, but as a person whose difficulties need to be understood in relation to events and how the person interpreted these events and reacted to them. ${ }^{9}$ Dynamic psychiatry furthermore addresses the fact of how a person continually recreates their internal conflicts in external situations; a patient can for example unconsciously recreate their internal world and their inner conflicts in the interpersonal field between them and their psychiatrist. Internal conflicts are then played out in the therapeutic relationship. ${ }^{11,12}$

The question in dynamic psychiatry is therefore how this patient differs from others with a similar descriptive diagnosis; who is this person, what makes him unique, what happened in their life, why does they present now with this problem, what is the meaning of this for them?' ${ }^{9}$ The information needed for this is gathered through the usual psychiatric interview, but also through the experience of being with the patient. The way patient and psychiatrist interact and how the psychiatrist feels during and after an interview can help them "to infer characteristic ways in which the patient responds to painful experiences and relates to others". ${ }^{9}$ It is however important to distinguish such feelings from counter-transference feelings having to do with the psychiatrist's own past and not to ascribe motives or attributes to the patient where these actually have to do with the doctor's past relationships and not with the patient. ${ }^{13}$ Counter-transference is nowadays generally regarded as the therapist's total emotional reaction to the patient, "entailing a jointly created reaction in the clinician that stems in part from contributions of the clinician's past and in part from feelings induced by the patient's behavior", 8,12

This information is then summarized in the psychodynamic formulation. This formulation is in dynamic psychiatry the real diagnosis; it refers to the understanding of the underlying dynamics of the maladaptive behavior and is thus etiological. ${ }^{7}$ The ability to formulate the problem of a patient in a psychodynamic way should be a key clinical skill for all psychiatrists. ${ }^{9}$ Vigorous research on the outcomes of psychodynamic therapy was until recently lacking. ${ }^{12}$ Limited confidence in the proven efficacy of the psychodynamic approach in psychiatry and inadequate exposure to psychodynamic theory in training programs could be reasons why a psychodynamic formulation is seldom offered or incorporated into the written record of the patient. ${ }^{14}$

\section{The psychodynamic formulation}

The psychodynamic formulation is a short description of the presenting problem in the context of the patient's life and includes non-dynamic as well as psychodynamic factors contributing to the current illness. It is drawn up after the assessment interview(s) and should focus on central conflicts, identified by pervasive issues that run through the patient's illness and can be traced back through his history. A thorough history, especially of the early years, is thus essential. The formulation should try to explain how the patient's attempts to resolve these central conflicts have been maladaptive, leading to symptoms, character pathology and problems in interpersonal relationships. ${ }^{14}$ 
Thinking in a psychodynamic way and creating such a formulation does not mean that the psychiatrist will necessarily work in a psychodynamic therapeutic way with the patient. The psychodynamic formulation is primarily an attempt to reach a better understanding of the patient, which then can be used in different ways; it can serve as a guide in the development of a treatment plan and to predict, for example, how the patient might interact with the psychiatrist or respond to being prescribed medication. ${ }^{14}$ It is meant as a guide for the therapist and not as something that should be directly interpreted to the patient. Although the psychodynamic formulation is only a hypothesis that will be tested and modified by additional data, it can be seen as the starting point in the interaction with the patient and is therefore of great importance.

\section{Common misperceptions about the psychodynamic formulation}

Common misconceptions are that a psychodynamic formulation is indicated only for patients in long-term psychoanalytic psychotherapy and that it is an unnecessary training exercise. It is however in any treatment important to understand the dynamics of the patient, in order to predict and manage the resistances the patient will present with and to design an approach that will circumvent these obstacles. ${ }^{14}$ Such a formulation is also of importance in patients where a biological approach will follow. ${ }^{9}$ A psychodynamic formulation should be written down, since the process of writing helps one achieve a clearer point of view. ${ }^{14}$

\section{The structure or format of a psychodynamic formulation}

There is no generally agreed format for a psychodynamic formulation. Some examples are so extensive that it can lead to feeling overwhelmed. Others remain essentially descriptive without giving a real psychodynamic explanation and prediction.

The following format, distilled from several sources, could

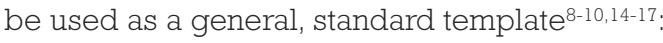

1. Construction of an illness narrative, consisting of a short identification of the patient, a summary of the presenting problem, followed by the most salient features of the patient's life history, for example childhood trauma, and at what stages in the patient's life major changes have occurred.

2. Identification of pathogenic factors:

2.1 Predisposing factors, for example a mother's lack of response to the needs of her child. Here the psychiatrist has to think in terms of developmental history.

2.2 Precipitating factors, for example a sense of abandonment brought about by marital problems. The focus here is on current life circumstances. 2.3 Maintaining factors:

- Internal: for example having internalized a tendency to be very critical of oneself. The personality organization is the focus.

- External: for example a difficult work environment. The psychiatrist has to look for triggers.
3. Assessment of non-dynamic factors that have contributed to the psychiatric disorder, for example a genetic predisposition, physical illness, socio-economic factors and cultural processes. The interplay of mind and brain in the development of mental illness is emphasized as well as the fact that a biologically driven symptom or illness may still have a psychodynamic meaning.

4. The psychodynamic explanation; the questions to be answered here are "Why does this person at this stage in his life suffer from this problem? How did it come about?" The explanation should include:

4.1 The personality structure with its different aspects such as:

- Control and regulation of instinctual (e.g. aggressive) drives

- Defense mechanisms used, e.g. primitive splitting or more mature suppression

- Capacity for interaction, communication and attachment

- Ability to empathize with others

- Self-perception and self-image

4.2 Central conflicts around the following themes ${ }^{9}$ :

- Dependency vs. autonomy

- Submission vs. control

- Desire for care vs. self-sufficiency

- Valuing self vs. valuing object

- Guilt conflicts

- Oedipal or sexual conflicts

- Identity conflicts

4.3 Characteristic patterns of interpersonal relations; the information for this coming from the history as well as the experience of being with the patient.

5. Prediction of the response to the therapeutic input. This part focuses on the meaning and use that the patient will make of treatment. Probable modes of resistance and manifestations of transference and counter-transference are postulated. Transference means that patterns of relatedness of the past are repeated in the present so that past experiences influence present relationships. , $^{8,13}$ The type and quality of the transference relationship is related to the quality of the primary relationships in the patient's life. Hence, in the relationship with the psychiatrist the transference could be seen as a new edition of an old pattern of relating that was established with the primary figures of the patient's childhood, namely with his parents.

Obviously not all of the aspects and themes mentioned in this format are applicable to a specific patient; these aspects and themes are only meant as a framework to guide the drawing up of a formulation and to stimulate thinking about the patient. It is also important to keep in mind that, apart from insight and understanding, many other factors play a role in healing and that the therapeutic alliance is the most crucial factor in determining the outcome of any psychotherapy. ${ }^{12}$ If at times a therapist feels lost in the complexity of the inner world of the patient, the words of the pediatrician and psychoanalyst Donald Winnicott (18961971) may provide solace: "I think I interpret mainly to let the patient know the limits of my understanding. The principle is that it is the patient and only the patient who has the answers." 18 


\section{A hypothetical example of a psychodynamic formulation \\ Illness narrative}

"P", 26 years of age and studying Christian counseling, presents with a history of recurrent depression. He recalls that he first suffered from a depressive episode about 10 years ago. At that stage he had an "identity crisis" when he thought that he might be gay. He tried to suppress such feelings and lead a "normal" life, but later admitted to himself and others that he was gay. Initially he had felt relieved, but in the last year he had become more and more depressed and had been treated with several antidepressants. His depression became worse in the last couple of weeks and he started to withdraw more and more from people.

His father, with whom he had a close relationship, died from a heart attack when Peter was in his first year at school. The mother, who is still alive, has a long-standing history of depression. He describes her as a withdrawn person; they never had a close relationship and he struggles with guilt feelings towards her, since he is still financially dependent on her. He has an older brother who is very successful in his career.

\section{Pathogenic factors}

The absent mother and early death of his father can be seen as predisposing factors. Early losses in childhood and problems in attachment lead to a vulnerability to depression in adulthood. ${ }^{8}$

A precipitating factor is the fear of soon having to start his career as a counselor in his church. His church does make allowance for homosexual counselors, but they have to live a life of celibacy. He states that such a life is a sacrifice God demands of him and claims that he has made peace with it.

Maintaining factors are guilt feelings towards his mother. He attributes the guilt feelings to still being dependent on her; it may however be that because of her depression she was often experienced in his infancy as being absent and not responding to his needs which could have led to an ambivalent identification with her and the development of aggressive feelings and therefore also guilt feelings towards her. This would contribute to the development of a harsh superego, which would exacerbate feelings of inferiority in relation to his very successful brother. The fact that he will soon have to start his career is also an ongoing stressor.

\section{Non-dynamic factors}

A family history of depression points towards the likely contribution of genetic factors in the etiology of his depression.

\footnotetext{
Psychodynamic explanation

- Personality structure

" $\mathrm{P}$ " is a sensitive and introverted person in whom the early death of his father and the problematic relationship with an absent or uninvolved mother played an important role in the development of his depression. His depressed mother could not respond to his needs and was experienced as a "bad object" leading to feelings of aggression towards her and insecure attachment.

Being however dependent on his mother, aggressive feelings were repressed and internalized and he developed a false, compliant self. An internal process of splitting led thus to
}

the identification with a bad internal object and dependency on external "good objects". The splitting was reinforced by the death of his father, which caused a regression and reawakening of oedipal conflicts, a conflict from which he, with the death of his father, emerged as the victor.

His main defense mechanisms at present are rationalization, intellectualization, repression and passivity.

\section{- Central conflicts}

The above-mentioned oedipal conflict, and the repressed anger towards the mother, led to severe unconscious guilt feelings and contributed to the development of a harsh superego. A healthy identification with a father figure who could withstand oedipal rivalry was not possible and this contributed to problems with his sexual identity.

It can also be postulated that the internalization of a "bad object", a sense of a bad self, as well as oedipal conflicts play a role in his passive acceptance of the demands of the church to lead a life of celibacy. Such an acceptance helps him to cope with his guilt feelings and also the underlying fear that rivalry can be destructive. After the second interview he mentioned a recent dream about someone having burnt himself; he however made a Freudian slip and said that the person "was a burnt offering"; the person was thus being sacrificed. This can be seen as an expression of his conflicts around submission and control and intense feelings about his view of having to obey the church and God, and thus be acceptable, and his desire to be himself, to be allowed to live his life and not to be sacrificed.

\section{- Interpersonal relationships}

His poor self-image also contributes to his depression. He is not assertive in his interactions with others and fears that he will be rejected, leading to further interpersonal problems and a withdrawal from friendships.

\section{Predicting responses to the therapeutic situation}

"P" will most likely welcome the option of psychodynamic insight oriented psychotherapy, because of his introspective nature and psychological mindedness. It can be expected that the patient will initially be very compliant and friendly towards his psychiatrist. He will probably, as part of the transference, see the psychiatrist in a similar way as a primary caretaking figure, whom he has to please and handle carefully. His aggressive, resentful self will be repressed and it will take some time to build enough confidence and trust to allow hidden feelings such as anger, resentment and rivalry to surface. He will then most probably become a more demanding and difficult patient who will test the ability of the psychiatrist to tolerate and contain these intense feelings. It is possible that with the deepening of the transference, anxiety and resistance may necessitate more supportive phases during psychotherapy, which may for example include cognitive or behavioral techniques.

\section{The advantages of incorporating a psychodynamic formulation}

According to DSM IV a patient like "P" would be diagnosed with a major depressive disorder, recurrent, moderate degree. Treatment with antidepressants in such a situation is accepted as good practice. A psychodynamic formulation highlights the 
internal conflicts and stressors endured; it indicates that, with a patient like "P", medication alone will not lead to remission and predicts that such a patient will be a good candidate for psychodynamic psychotherapy. According to the type of disorder and personality of the patient, as well as other factors such as the expertise available, other forms of therapy, for example cognitive-behavior therapy could be indicated.

\section{Conclusion}

The aim of dynamic psychiatry is not to replace descriptive psychiatry and the descriptive diagnosis, but rather to promote greater sensitivity towards patients and to recognize the continuity between the inner life of the patient and his mental problems. ${ }^{15}$ Dynamic psychiatry requires of the psychiatrist a willingness to enter into the patient's life and experiences and to see problematic behavior as an outflow of unmanageable feelings and conflicts. The psychodynamic formulation, being a short description of the unique inner life of the patient, plays an important role in dynamic psychiatry since it can help anticipate a patient's response to treatment and thus allows for better management of patients. The ability to formulate the problems of a patient in a psychodynamic way should be an integral part of psychiatric training. ${ }^{15}$ The formulation should not be overestimated, but seen as a tentative attempt to make sense of the patient's emotional problems and to provide initial guidance. The importance of entering into the uniqueness of each patient's life cannot however be overestimated.

\section{Acknowledgement}

The author thanks Ms J. Blom, clinical psychologist, for her helpful comments.

\section{References}

1. Jamison KR. An Unquiet Mind. London: Picador, 1997: 88-89.

2. Kendler KS. Toward a philosophical structure for psychiatry. Am J Psychiatry 2005; 162: 433-440

3. Kandel ER. A new intellectual framework for psychiatry. Am J Psychiatry 1998; 155(4): 457-469.

4. Kandel ER. Biology and the future of psychoanalysis: A new intellectual framework for psychiatry revisited. Am J Psychiatry 1999;
156(4): 505-524

5. Browning D. Internists of the mind or physicians of the soul: Does psychiatry need a public philosophy? Zygon: J of Religion and Science 2008; 43(2): 371-383

6. Josephson AM, Peteet JR. Talking with patients about spirituality and worldview: Practical interviewing techniques and strategies. Psychiatric Clinics of North America 2007; 30(2): 181-197.

7. Wallace ER. Dynamic Psychiatry in Theory and Practice. Philadelphia: Lea \& Febiger, 1983: 1-4

8. Gabbard GO. Psychodynamic Psychiatry. 4th Edition. Arlington: American Psychiatric Publishing, 2005: 4-7, 21 1-221.

9. Mace C, Binyon S. Teaching psychodynamic formulation to psychiatric trainees. Part 1: Basics of formulation. Advances in Psychiatric Treatment 2005; 11:416-423

10. Aveline M. The advantages of formulation over categorical diagnosis in explorative psychotherapy and psychodynamic management. European J of Psychotherapy, Counseling and Health 1999; 2(2): 199-216.

11. Ogden TH. The Matrix of the Mind: Object relations and the Psychoanalytic Dialogue. Northvale, New Jersey: Jason Aronson, 1990: 131-165

12. Gabbard GO. Long-term Psychodynamic Psychotherapy. A Basic Text. 2nd Edition. Washington, DC: American Psychiatric Publishing, 2010: 53

13. Böhmer MW. "Communication by impact" and other forms of nonverbal communication: A review of transference, counter-transference and projective identification. Afr J Psychiatry 2010; 13: 179-183.

14. Perry S, Cooper AM, Michels R. The psychodynamic formulation: Its purpose, structure, and clinical application. Am J Psychiatry 1987; 144: 543-550

15. Mace C, Binyon S. Teaching psychodynamic formulation to psychiatric trainees. Part 2: Teaching methods. Advances in Psychiatric Treatment 2006; 12: 92-99.

16. Kassan K, Gabbard GO. Creating a psychodynamic formulation from a clinical evaluation. Am J Psychiatry 2002; 159(5): 721-726.

17. Bateman A, Brown D, Pedder J. Introduction to Psychotherapy. An Outline of Psychodynamic Principles and Practice. 4th Edition. London: Routledge, 2010: 239-254

18. Winnicott D. Playing and Reality. London: Reprinted Routledge Classics, 2005: 116

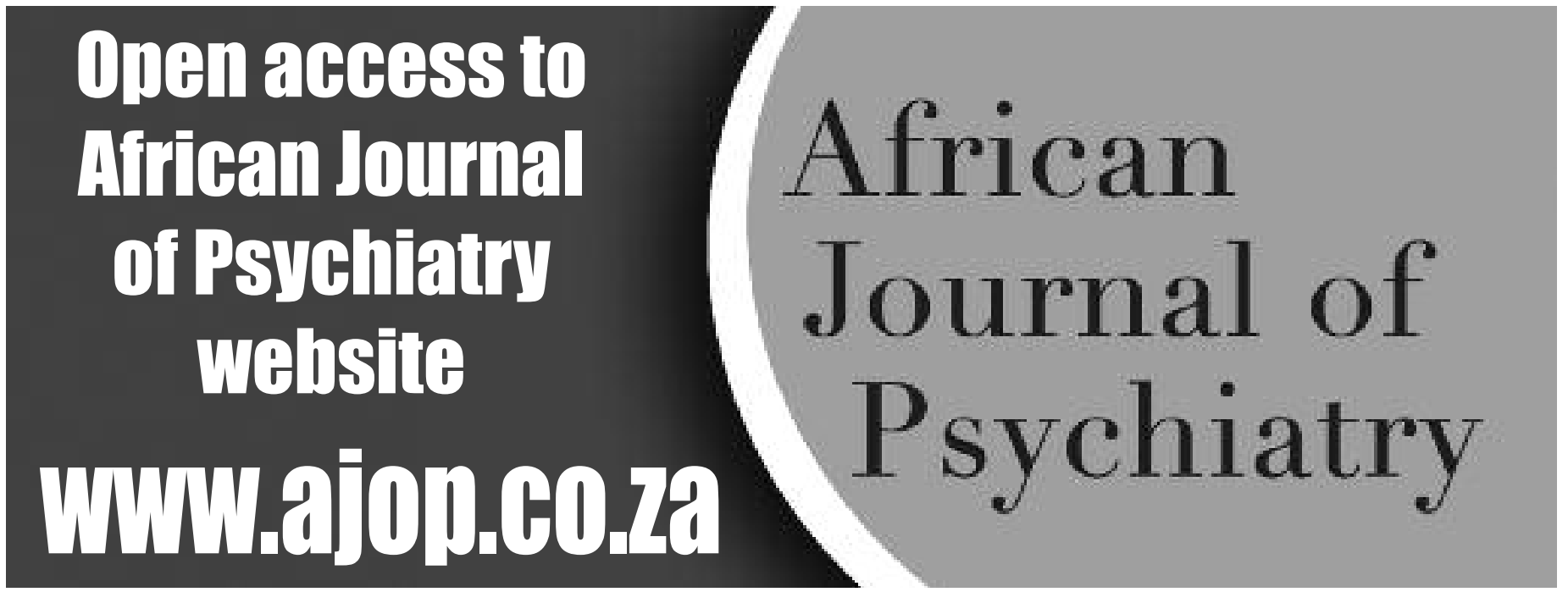

